\title{
$2+2$ GNYERATIONAL IOURNAL OF \\ Surgical staging, the meaning of life, and other existential ponderings
}

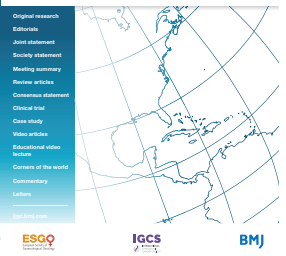

Michael Frumovitz

Correspondence to Dr Michael Frumovitz, Department of Gynecologic Oncology and Reproductive Medicine, University of Texas MD Anderson Cancer Center, Houston, TX 77030, USA; mfrumovitz@mdanderson.org

Received 21 0ctober 2020 Accepted 21 October 2020

\section{Sinked}

- http://dx.doi.org/10.1136/ ijgc-2020-001973

\section{Check for updates}

(C) IGCS and ESGO 2020. No commercial re-use. See rights and permissions. Published by BMJ.

To cite: Frumovitz M. Int J Gynecol Cancer 2020;30:1862-1863.
Countless academics have contemplated life's many unanswered questions. What existed before the universe? Will we ever discover life on other planets? Does free will truly exist? Why does a round pizza come in a square box? For years gynecologic and radiation oncologists have pondered the question of surgical staging for women with locally advanced cervical cancer (stages IB3-IVA). Does pathologic assessment of para-aortic lymph nodes improve survival by extending the radiation field in patients with microscopically positive para-aortic nodes while decreasing morbidity by limiting the radiation field in those patients who are negative for metastases?

The rationale for surgical staging stems from the inadequacies of current radiologic modalities to detect metastatic disease. MRI has a sensitivity of only $38 \%$ in detecting lymph node metastases while CT scan performs somewhat better, finding metastatic disease in lymph nodes $67 \%$ of the time. ${ }^{1}$ More recently, fluorodeoxyglucose (FDG)-positron emission tomography (PET) has been commonly used in the initial work-up of patients with newly diagnosed cervical cancer as PET scans have a sensitivity of $84 \%$ in identifying metastatic disease in para-aortic lymph nodes. ${ }^{23}$ In other words, even with our best imaging modality, PET scan, $16 \%$ of women with locally advanced cervical cancer will have undetected disease in paraaortic nodes, probably leading to under-treatment, with radiation fields limited to the pelvis. One would assume that surely this equates with increased recurrence and decreased survival for these patients.

Retrospective studies on this topic, however, have been anything but conclusive. Early studies showed surgical staging detects more disease in nodes than imaging and but failed to show that finding this microscopic disease changed survival. ${ }^{45}$ However, more recently larger retrospective studies have shown a survival benefit from surgical staging compared with clinical and radiologic staging. Gold et $\mathrm{al}^{6}$ evaluated 685 patients who had participated in three Gynecologic Oncology Group (GOG) chemoradiation studies. In two of those studies, surgical staging was mandatory and in the third it was optional. In comparison with patients who had only radiologic staging $(n=130)$, women who underwent surgical staging $(n=555)$ had significantly better 4-year progression-free survival
( $36 \%$ vs $49 \%$ ) and overall survival ( $40 \%$ vs $54 \%$ ). With data like these in mind, many have been optimistic that a well-designed, prospective clinical trial would finally show that surgical staging improves outcomes for women with locally advanced cervical cancer.

In this issue of the International Journal of Gynecological Cancer, we are honored to publish the muchanticipated results of Uterus-11. ${ }^{7}$ The investigators must first be commended on completing a prospective, randomized clinical trial of surgical staging in patients with locally advanced cervical cancer when other attempts were closed well before accrual was completed. ${ }^{89}$ Although the trial did not show a difference between the two arms in progression free or overall survival (we will get to that below), they did prove other important outcomes. First, they demonstrate a trial for surgical staging in patients with locally advanced cervical cancer is feasible and can be completed in a reasonable period of time. Second, they prove that pre-therapeutic surgical staging does not significantly delay initiation of definitive chemoradiation. Third, in line with previously published reports, the study confirms a high rate of upstaging of disease, with $33 \%$ of patients who undergo surgical staging having microscopic metastatic disease in lymph nodes detected that otherwise would not have been found on a pre-therapeutic CT scan. Finally, they show that laparoscopic staging using a transperitoneal approach does not significantly increase radiation toxicity. This is an important outcome as many have argued against surgical staging because of concerns about increasing long-term morbidity of radiation when administered after surgical exploration.

Although all of these outcomes are favorable for surgical staging, the most important endpoints of improving progression free and overall survival were not met. There was no difference between the two arms in progression free survival, the primary endpoint, and although the hazard ratio for recurrence was 0.71 favoring surgical staging and although this did not meet significance, the $p$ value of 0.08 certainly showed a trend towards supporting surgery. The secondary endpoint of overall survival was also not statistically different between the two groups, but again the hazard ratio for death of 0.69 $(p=0.07)$ showed a trend towards favoring surgery in 
these patients. The authors performed post hoc analyses and were able to show that surgery improves cancer-specific survival as well as improving survival for patients with stage IIB disease. Although these are interesting, hypothesis-generating outcomes, there is no hiding the fact that this is a negative study.

So, have we finally answered the age-old question of surgical staging for locally advanced cervical cancer? l'm not so sure. The fatal flaw of this study, I believe, is in the sample size calculation (admittedly it is easy to criticize in hindsight decisions made many years ago). The authors estimated a 5-year-disease free survival of $36.5 \%$ for the control arm of clinical staging. In the era of chemoradiation, a more reasonable recurrence rate in patients with locally advanced cervical cancer (stages IB3-IVA) staged clinically/radiologically is probably closer to $50-60 \% .{ }^{10}$ In fact, in this study (Uterus-11) the recurrence rate in the group that was clinically staged was $55 \%$. The experimental arm (surgery), on the other hand, was estimated to improve 5-year disease-free survival by $50 \%$. This is an ambitious estimate when surgical staging has traditionally found only an additional $33 \%$ of patients with unrecognized disease in para-aortic nodes compared with a CT scan. ${ }^{1}$ With more accurate estimates of disease-free survival in clinically staged patients and more modest predictions of how much surgical staging would improve survival, sample sizes would be much larger and the trends towards progression-free and overall survival seen in this study would probably become statistically significant. If that were the case, we might be celebrating a positive, practicechanging study. This study demonstrates the importance of making realistic assumptions when calculating the sample size as opposed to choosing the sample size and then 'massaging' the data to arrive at the sample size one wants to obtain.

So, where do we go from here? I do not believe we should abandon the concept of pre-therapeutic surgical staging for patients with locally advanced cervical cancer. As this study shows, transperitoneal surgical staging for these patients is safe and does not delay the start of definitive chemoradiation nor does it increase radiation morbidity. Second, a study with adequate sample size is likely to show an improvement in survival for surgically staged patients. I would strongly encourage any collaborative group or groups that have the funding, the will, and the patient volume to consider an adequately powered study comparing surgical staging with PET staging in patients with locally advanced cervical cancer.

And now onto other important questions like, do penguins have knees?

Twitter Michael Frumovitz @frumovitz

Funding The author has not declared a specific grant for this research from any funding agency in the public, commercial or not-for-profit sectors.

Competing interests None declared.

Patient consent for publication Not required.

Provenance and peer review Commissioned; internally peer reviewed.

\section{REFERENCES}

1 Camilien L, Gordon D, Fruchter RG, et al. Predictive value of computerized tomography in the presurgical evaluation of primary carcinoma of the cervix. Gynecol Oncol 1988;30:209-15.

2 Havrilesky LJ, Kulasingam SL, Matchar DB, et al. FDG-PET for management of cervical and ovarian cancer. Gynecol Oncol 2005;97:183-91.

3 Ramirez PT, Jhingran A, Macapinlac HA, et al. Laparoscopic extraperitoneal para-aortic lymphadenectomy in locally advanced cervical cancer: a prospective correlation of surgical findings with positron emission tomography/computed tomography findings. Cancer 2011;117:1928-34.

4 LaPolla JP, Schlaerth JB, Gaddis O, et al. The influence of surgical staging on the evaluation and treatment of patients with cervical carcinoma. Gynecol Oncol 1986;24:194-206.

5 Podczaski ES, Palombo C, Manetta A, et al. Assessment of pretreatment laparotomy in patients with cervical carcinoma prior to radiotherapy. Gynecol Oncol 1989;33:71-5.

6 Gold MA, Tian C, Whitney CW, et al. Surgical versus radiographic determination of para-aortic lymph node metastases before chemoradiation for locally advanced cervical carcinoma: a gynecologic Oncology Group study. Cancer 2008;112:1954-63.

7 Marnitz S, Tsunoda AT, Martus P. Surgical versus clinical staging prior to primary chemoradiation in patients with cervical cancer FIGO stages IIB-IVA: oncologic results of a prospective randomized international multicenter (Uterus-11) intergroup study. Int J Gynecol Cancer 2020.

8 Lai C-H, Huang K-G, Hong J-H, et al. Randomized trial of surgical staging (extraperitoneal or laparoscopic) versus clinical staging in locally advanced cervical cancer. Gynecol Oncol 2003;89:160-7.

9 Frumovitz M, Querleu D, Gil-Moreno A, et al. Lymphadenectomy in locally advanced cervical cancer study (LiLACS): phase III clinical trial comparing surgical with radiologic staging in patients with stages IB2-IVA cervical cancer. J Minim Invasive Gynecol 2014;21:3-8.

10 Chemoradiotherapy for Cervical Cancer Meta-analysis Collaboration (CCCMAC). Reducing uncertainties about the effects of chemoradiotherapy for cervical cancer: individual patient data metaanalysis. Cochrane Database Syst Rev 2010:CD008285. 